\title{
Inhibition of c-MET is a potential therapeutic strategy for treatment of diffuse large B-cell lymphoma
}

\author{
Shahab Uddin ${ }^{1,3}$, Azhar R Hussain ${ }^{1,3}$, Maqbool Ahmed ${ }^{1}$, Fouad Al-Dayel ${ }^{2}$, Rong Bu ${ }^{1}$, Prashant Bavi ${ }^{1}$ and \\ Khawla S Al-Kuraya ${ }^{1}$
}

Hepatocyte growth factor/c-MET has emerged as a potential therapeutic target for several cancers; however, its role in diffuse large B-cell lymphoma (DLBCL) has not been fully elucidated. In this study, we first investigated the role of c-Met in a large series of DLBCL tissues in a tissue microarray format. We then followed this with in vitro studies on DLBCL cell lines using either pharmacological inhibitors of c-Met or siRNA knockdown strategy. c-Met was found to be overexpressed in $73.2 \%$ of patients $(186 / 254)$ and was significantly associated with overexpression of p-AKT $(P=0.0274)$, p-GSK3 $(P=0.0047)$ and $\mathrm{Ki}-67(P=0.0012)$. Interestingly, $\mathrm{c}-$ Met overexpression was significantly more common in the germinal center subtype of DLBCL, as compared with activated $\mathrm{B}$ cell subtype $(P=0.0002)$. Overexpression of $\mathrm{C}-\mathrm{Met}$ in DLBCL was significantly associated with better survival $(P=0.0028)$ and remained significant in multivariate analysis with international prognostic index, thereby confirming c-Met as independent prognostic marker for better outcome in DLBCL. In vitro pharmacological c-Met inhibition and siRNA targeted against c-Met triggered caspase-dependent apoptosis. These findings provide evidence that c-Met is an independent prognostic marker for better outcome in Middle Eastern DLBCL. This data also enlightens the fact that c-Met through AKT kinase has a critical role in carcinogenesis of DLBCL, and strongly suggest that targeting c-Met may have therapeutic value in treatment of DLBCL.

Laboratory Investigation (2010) 90, 1346-1356; doi:10.1038/labinvest.2010.108; published online 7 June 2010

KEYWORDS: c-Met; AKT; apoptosis; DLBCL

B-cell lymphoma represents the malignant counterpart of normal $\mathrm{B}$ cells arrested at specific maturational stages. Diffuse large B-cell lymphoma (DLBCL) is considered to be the most common type of lymphoma in adults, accounting for $30-40 \%$ of cases of non-Hodgkin lymphoma. ${ }^{1}$ Although patients with DLBCLs are potentially curable with combination chemotherapy, the disease proves fatal in $\sim 50 \%$ of patients. ${ }^{2}$ The cause of most DLBCLs remains unknown; however, dysregulation of apoptosis or defective repair has a role in lymphogenesis. ${ }^{3}$

A number of activated growth factor signaling pathways have frequently been observed in DLBCL, including hepatocyte growth factor (HGF)/MET pathway. ${ }^{4,5}$ The HGF receptor, c-MET, is a tyrosine kinase receptor with established oncogenic properties. Activation of c-MET results in phosphorylation that leads to recruitment of adaptor proteins and to the subsequent activation of various signal transducers including phosphatidylinositol 3-kinase (PI3K) and extracellular regulated kinase, ultimately resulting in stimulation of growth, survival, motility and invasion of cancer cells. ${ }^{6}$ Although c-MET is largely expressed on epithelial cells, certain cells of hematopoietic origin also carry this receptor and are sensitive to HGF. For example, c-MET is found on germinal center B cells and on terminally differentiated plasma cells. ${ }^{5,7}$ The receptor also has a role in some hematological malignancies, for example, primary effusion lymphoma, multiple myeloma and, notably, DLBCL. ${ }^{8-10}$

Previously, overexpression of either HGF or c-MET in DLBCL tumor cells, as well as high serum level of HGF in DLBCL patients, has been found to be associated with poor prognosis. ${ }^{11-13}$ More recently, a functional analysis study has shown that HGF/MET signaling has an important role in the maintenance of DLBCL in the tumor microenvironment. ${ }^{10}$

\footnotetext{
${ }^{1}$ Human Cancer Genomic Research, Research Center, King Faisal Specialist Hospital and Research Center, Riyadh, Saudi Arabia and ${ }^{2}$ Department of Pathology,

King Faisal Specialist Hospital and Research Center, Riyadh, Saudi Arabia

Correspondence: Dr KS Al-Kuraya, MD, FCAP, Human Cancer Genomic Research, Research Center, King Faisal Specialist Hospital and Research Cancer, MBC\#98-16, P.O. Box 3354, Riyadh 11211, Saudi Arabia.

E-mail: kkuraya@kfshrc.edu.sa

${ }^{3}$ These are the joint first authors of this article. 
Altogether, these studies suggest that c-MET may be an attractive candidate for targeted therapy in DLBCL.

In the present study, we first analyzed the prevalence of c-Met overexpression in $>300$ Middle Eastern DLBCL patients' samples and their clinicopathological association and prognostic significance by tissue microarray (TMA). Then we investigated the effects of pharmacological inhibition of c-Met on DLBCL cell viability, apoptosis and downstream signaling pathways.

\section{MATERIALS AND METHODS Construction of TMA}

Tissue microarrays were constructed, as described previously. ${ }^{14}$ One pathologist $(\mathrm{PB})$ reviewed all DLBCL slides and mapped the representative non-necrotic tumor areas. Briefly, tissue cylinders with a diameter of $0.6 \mathrm{~mm}$ were punched from 301 DLBCL patients' archival paraffin blocks and transferred into a recipient paraffin block using a semi automatic precision instrument (Beecher Instruments, Silver Spring, MD, USA).

\section{Patient Samples}

A total of 301 cases of de novo DLBCL diagnosed between 1987 and 2006 and reclassified according to WHO criteria, ${ }^{15}$ were collected from the Department of Pathology at King Faisal Specialist Hospital and Research Centre (KFSH\&RC). Archival paraffin blocks and clinical data were obtained by reviewing the charts, according to the regulations of the institutional review board of the hospital. Patients were staged by means of computerized imaging and bone marrow biopsy. The institutional review board of KFSH\&RC approved the study.

\section{Immunohistochemistry}

Tissue microarray slides were processed and stained manually. The immunohistochemistry (IHC) protocol was followed as mentioned earlier. ${ }^{16}$ Primary antibodies used, their dilutions and cutoff levels for evaluation are listed in Supplementary Table 1 . Only fresh-cut slides were stained simultaneously to minimize the influence of slide ageing and maximize reproducibility of the experiment.

\section{IHC assessment}

c-Met expression was categorized by doing $H$-score. ${ }^{17}$ Each TMA spot was assigned an intensity score from 0 to 3 (I0, I1-3), and proportion of the tumor staining for that intensity was recorded as 5\% increments from a range of 0-100 (P0, P1-3). A final $H$-score (range $0-260$ ) was obtained by adding sum of scores obtained for each intensity and proportion of area stained $(H$-score $=\mathrm{I} 1 \mathrm{XP} 1+\mathrm{I} 2 \mathrm{XP} 2+\mathrm{I} 3 \mathrm{XP} 3)$. X-tile plots were constructed for assessment of biomarker and optimization of cutoff points on the basis of outcome, as has been described earlier. ${ }^{18}$ p-AKT and PTEN scoring was carried out as described earlier. ${ }^{19,20}$ The expression of CD10, BCL6 and
MUM1 was considered to classify tumors as germinal center (GC) B-cell-like DLBCL and activated B cell (ABC), applying the decision tree described earlier. ${ }^{21,22} \mathrm{X}$-tile plots were constructed for assessment of biomarker and optimization of cutoff points on the basis of outcome, as has been described earlier. $^{23}$

\section{Statistical analysis}

The software used for statistical analysis was Statview 7.0 (SAS Institute, NC, USA). The $\chi^{2}$-tests were used to examine relationship between nominal variables. The limit of significance for all analyses was defined as a $P$-value of 0.05 ; two-sided tests were used in all calculations.

Isolation of peripheral blood mononuclear cells from healthy donors

Peripheral blood mononuclear cells (PBMNCs) were isolated using ficoll-hypaque gradient centrifugation. Blood from healthy donors were diluted in the ratio 1:1 with $1 \times$ PBS and equal amount of ficoll was added to blood. The samples were spun at 3000 r.p.m. for $30 \mathrm{~min}$ and buffy coat was isolated, washed with PBS and treated with PHA665752 for $24 \mathrm{~h}$, and cells were analyzed by flow cytometry.

\section{Cell culture}

The human DLBCL cell lines, SUDHL4, SUDHL5 and SUDHL10, were obtained from Deutsche Sammlung von Mikroorganismen und Zellkulturen (DSMZ), Braunschweig, Germany. All cell lines were tested for immunological markers and cytogenetics. The cell lines were also fingerprinted and species were confirmed by IEF of AST, MDH and NP. SUDHL4 and SUDHL10 have a germinal center-like phenotype, whereas SUDHL5 has an ABC-like phenotype. The cell lines were cultured in RPMI 1640 medium supplemented with $20 \%(\mathrm{v} / \mathrm{v})$ fetal bovine serum, $100 \mathrm{U} / \mathrm{ml}$ penicillin, $100 \mathrm{U} / \mathrm{ml}$ streptomycin at $37^{\circ} \mathrm{C}$ in a humidified atmosphere containing $5 \% \mathrm{CO}_{2}$. All the experiments were performed in RPMI-1640 medium containing 5\% serum.

\section{Reagents and Antibodies}

PHA665752 were purchased from Tocris Bioscience (Ellisville, MO, USA). Bax (6A7) antibody was purchased from Sigma (St Louis, MO, USA). Antibodies against p-AKT, caspase-9, p-FOXO-1, p-GSK3, cleaved caspase-3 and Bid were purchased from Cell Signaling Technologies (Beverly, MA, USA). c-Met and phosphorylated MET (p-Met) antibodies were purchased from Biosource, (Camarillo, CA, USA). Cytochrome $c, \beta$-actin, caspase- 3 and PARP antibodies were purchased from Santa Cruz Biotechnology (Santa Cruz, CA, USA). XIAP, cIAP-1, Survivin and caspase-8 antibodies were purchased from R\&D (Minneapolis, MN, USA). Annexin-V was purchased from Molecular Probes (Eugene, OR, USA). Apoptotic DNA-ladder kit was obtained from Roche (Penzberg, Germany). 


\section{Cell cycle analysis, annexin-V staining and DNA laddering}

Diffuse large B-cell lymphoma cell lines were treated with different concentrations of PHA665752, as described in the legends. For cell cycle analysis, cells were washed once with PBS and resuspended in $500 \mu \mathrm{l}$ hypotonic staining buffer and analyzed by flow cytometry, as described previously. ${ }^{24-26}$ For detection of apoptosis, cells were harvested and percentage apoptosis was measured by flow cytometry after staining with flourescein-conjugated annexin- $\mathrm{V}$ and propidium iodide (PI) (Molecular probes) and DNA laddering using a 1.5\% agarose gel, as described previously. ${ }^{27}$

\section{Cell lysis and immunoblotting}

Cells were treated as described in the legends and lysed as previously described. ${ }^{28}$ A measure of $15-20 \mu$ g of proteins were separated by SDS-PAGE and transferred to polyvinylidene difluoride membrane (Immobilion; Millipore, Bellerica, MA, USA). Proteins were immunoblotted with different antibodies and visualized by enhanced chemiluminescence (Amersham, Piscataway, NJ, USA).

\section{Measurement of mitochondrial potential using the JC1}

In total, $1 \times 10^{6}$ cells were treated with PHA665752 for $24 \mathrm{~h}$. Cells were washed with PBS and suspended in mitochondrial incubation buffer (Alexis, Farmingdale, NY, USA). JC1 (5,5',6,6'-tetrachloro- $1,1^{\prime}, 3,3^{\prime}$-tetraethylbenzimidazolylcarbocyanine iodide) was added to a final concentration of $10 \mu \mathrm{M}$ and cells were incubated at $37^{\circ} \mathrm{C}$ in dark for $15 \mathrm{~min}$. Cells were washed twice with PBS and resuspended in $500 \mu \mathrm{l}$ of mitochondrial incubation buffer, and mitochondrial membrane potential (percentage of green and red aggregates) was determined by flow cytometry, as described previously. ${ }^{29}$

\section{Assay for cytochrome c release}

Release of cytochrome $c$ from mitochondria was assayed as described earlier. ${ }^{17}$ A measure of $15-20 \mu$ g proteins from cytosolic fraction, as well as mitochondrial fraction, of each sample were analyzed by immunoblotting using an anticytochrome $c$ antibody.

\section{Detection of Bax conformational changes}

Cells were treated with indicated doses of PHA665752, lysed with Chaps lysis buffer (10 mM HEPES (pH 7.4), $150 \mathrm{mM}$ $\mathrm{NaCl}, 1 \%$ Chaps) and immunoprecipitated with anti-Bax $6 \mathrm{~A} 7$ monoclonal antibody; Bax conformation was detected as described earlier. ${ }^{25,27}$

\section{Gene silencing using siRNA}

c-Met siRNA was purchased from Santa Cruz Biotechnology. AKT siRNA, AKT siRNA and scrambled control siRNA were purchased from Qiagen (Valencia, CA, USA). Cells were transfected using Lipofectamine 2000 (Invitrogen, Carlsbad, CA, USA), as described earlier. ${ }^{30}$ After $6 \mathrm{~h}$ of transfection, lipid and siRNA complex was removed and fresh growth medium was added. Cells were lysed $48 \mathrm{~h}$ after transfection and specific protein levels were determined by western blot analysis with specific antibodies.

\section{Statistical analysis}

Data are presented as mean \pm s.d. Comparisons between groups were made with the paired Student's $t$-test. Values of $P<0.05$ were considered statistically significant.

\section{RESULTS \\ c-Met Expression and its Correlation with p-AKT and Other Clinicopathological Parameters}

High levels of c-Met expression were seen in 73.2\% (186/254) of the DLBCL (Figure 1A). As shown in Table 1, c-Met overexpression was significantly associated with overexpression of p-AKT $(P=0.0274), \mathrm{p}$-GSK3 $(0.0047)$ and Ki-67 $(P=0.0012)$ (Table 1). High level of c-Met expression was also significantly associated with expression of PTEN $(P=0.0182)$. Interestingly, c-Met overexpression was significantly more common in the GC subtype of DLBCL, as compared with $\mathrm{ABC}$ subtype $(P=0.0002)$ However, c-Met overexpression was not associated with age, sex, stage, $\mathrm{LDH}$ level and international prognostic index (IPI). Intriguingly, DLBCL patients with overexpression of c-Met had a better overall survival of $76.2 \%$ at 5 years, as compared with $57.5 \%$ $(P=0.0028)$ with low c-Met expression. In the multivariate analysis using Cox proportional hazard model for both factors, IPI and c-Met, the relative risk was 2.33 for low c-Met expression (95\% CI 1.12-4.70; $P=0.0241)$ and 4.30 for high-risk IPI group (95\% CI 2.11-9.14; $P=<0.0001$ ). Thus, c-Met overexpression was an independent prognostic marker for better survival in Middle Eastern DLBCL patients (Supplementary Table 2).

\section{c-Met Inhibition Causes Cell Death and Induces Apoptosis in DLBCL Cell Lines}

We next sought to determine whether PHA665752, a specific inhibitor of c-Met treatment, leads to cell death in DLBCL cell lines. SUDHL4, SUDHL5 and SUDHL10 cells were cultured in the presence or absence of 0,1 and $5 \mu \mathrm{M}$ for $24 \mathrm{~h}$, and cells were stained with trypan blue. Manual counts showed that SUDHL4, SUDHL5 and SUDHL10 cell lines underwent cell death in a dose-dependent manner after treatment with PHA665752 (Figure 2a). To confirm these results, cell cycle fractions were determined by flow cytometry after treatment with 1 and $5 \mu \mathrm{M}$ PHA665752 in all three DLBCL cell lines. As shown in Figure 2b, the sub-G1 population of SUDHL4 cells was increased from $0.42 \%$ in the control to $9.44 \%$ and $73.04 \%$ at 1 and $5 \mu \mathrm{M}$ PHA665752, respectively. Similarly, Apo fraction increased in SUDHL5 and SUDHL10 cell lines in a dose-dependent manner. It has been reported that cells with these features are those dying of apoptosis. ${ }^{24-26}$ To further confirm that this increase in the sub-G1 population in DLBCL cell lines was indeed apoptosis, DLBCL cells were treated with 1 and $5 \mu \mathrm{M}$ PHA665752 and apoptotic cells were assayed by Annexin-V/PI dual staining. 

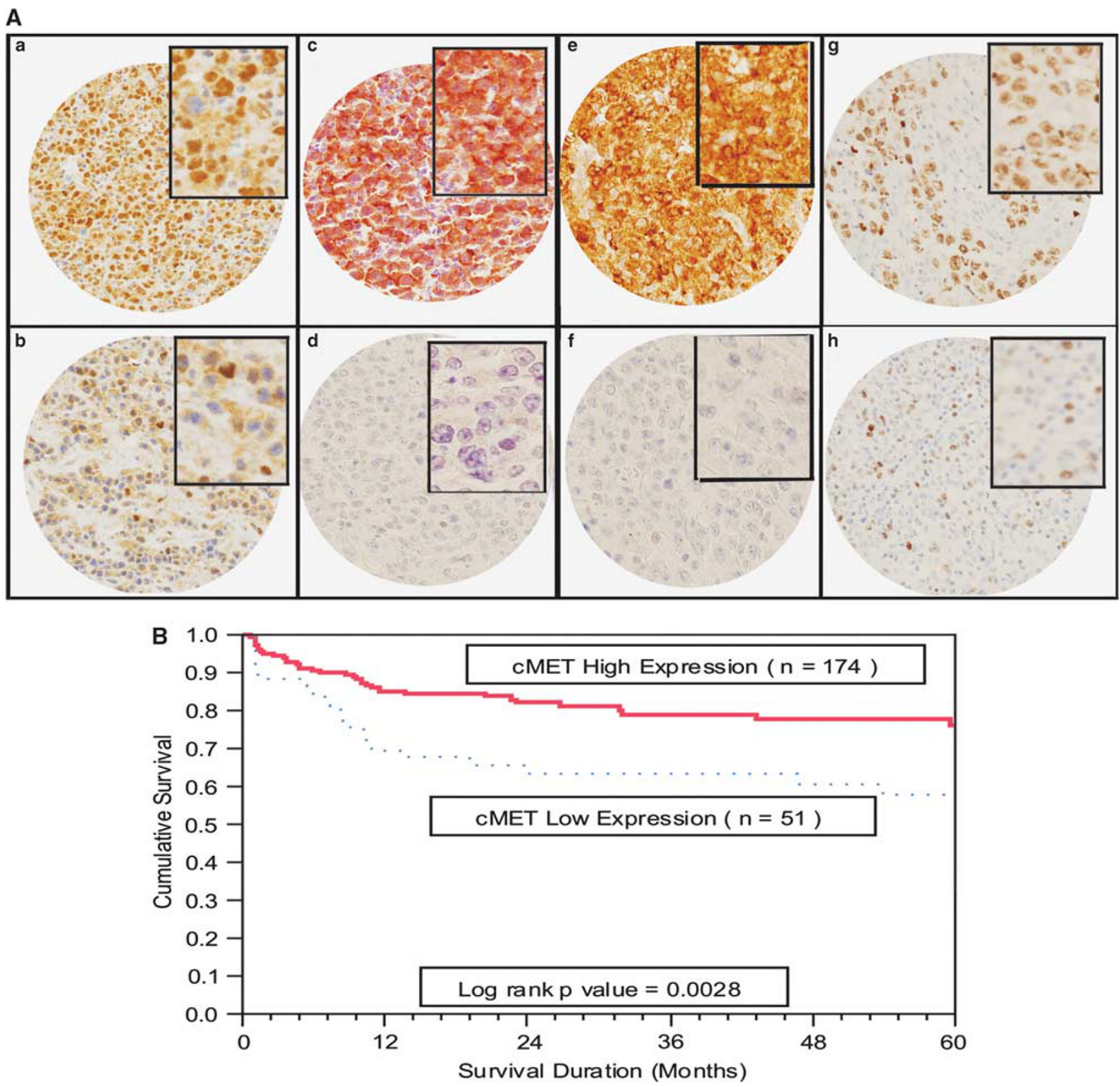

Figure 1 (A) Tissue microarray-based immunohistochemical analysis of c-Met, p-AKT, p-GSK3 and Ki67 expression in DLBCL patients. (A) (a) c-Met overexpression was observed along with (c) overexpression of p-AKT (e) p-GSK3 and (g) Ki67 in DLBCL TMA specimen. (b) Decreased staining for c-Met was seen along with (d) low expression of p-AKT, (f) p-GSK3 and (h) Ki67 in another DLBCL TMA specimen. $20 \times$ magnifications with the inset showing a $100 \times$ magnified view of the same. (B) Impact of c-Met expression on prognosis in DLBCL patients. Patients with overexpression of c-Met had a good overall survival of $76.2 \%$ at 5 years $(n=174, P=0.0028)$, as compared to $57.5 \%$ with low c-Met expression $(n=51)$.

As shown in Figure 2c, treatment with PHA665752 of SUDHL4, SUDHL5 and SUDHL10 cells resulted in apoptosis, as detected by annexin-V/PI dual staining. In addition, we also treated normal PBMNCs from five healthy donors with 1 and $5 \mu \mathrm{M}$ PHA665752 for $24 \mathrm{~h}$. No appreciable amount of apoptosis was detected by annexin-V/PI dual staining (Figure 2d). Finally, we confirmed the response of DLBCL cell lines to PHA665752 by DNA laddering. We treated SUDHL4 and SUDHL5 cell lines with 25 and $50 \mu \mathrm{M}$
PHA665752 for $24 \mathrm{~h}$ and detected DNA fragmentation in both the cell lines (Figure 2e). These data indicate that inhibition of c-Met kinase activity resulted in induction of apoptosis in DLBCL cell lines.

\section{Constitutive Activation of c-Met/AKT Signaling Pathway in DLBCL Cell Lines}

Activation of c-Met receptor occurs through its phosphorylation, which leads to modulation of various signaling 
Table 1 Clinical characteristics and CMET (C and N) status of patients with DLBCL

\begin{tabular}{|c|c|c|c|c|c|c|c|}
\hline \multirow{2}{*}{$\begin{array}{l}\text { DLBCL-301 } \\
\text { August } 2008\end{array}$} & \multicolumn{2}{|c|}{ Total } & \multicolumn{2}{|c|}{ Any-one-high } & \multicolumn{2}{|c|}{ Both low } & \multirow[t]{2}{*}{$P$-value } \\
\hline & No. & $\%$ & No. & $\%$ & No. & $\%$ & \\
\hline
\end{tabular}

$\begin{array}{lrrrrrrr}\text { No. of patients } & 254 & & 186 & 73.2 & 68 & 26.8 & \\ & & & & & & & \\ \text { Age (all patients) } & & & & & & & \\ \quad \leqslant 60 & 167 & 65.7 & 124 & 74.2 & 43 & 25.8 & 0.6111 \\ >60 & 87 & 35.3 & 62 & 71.3 & 25 & 28.7 & \end{array}$

$\begin{array}{lrrrrrrr}\text { Sex (all patients) } & & & & & & & \\ \text { Female } & 106 & 41.7 & 82 & 77.4 & 24 & 22.6 & 0.2057 \\ \text { Male } & 148 & 58.3 & 104 & 70.3 & 44 & 29.7 & \end{array}$

$\begin{array}{lrllllll}\text { Performance status (IPI) } & & & & & & & \\ 0-2 & 100 & 59.5 & 77 & 77.0 & 23 & 23.0 & 0.8861 \\ 3-4 & 68 & 40.5 & 53 & 77.9 & 15 & 22.1 & \end{array}$

$\begin{array}{llllllll}\text { Stage (IPI) } & & & & & & & \\ \text { I-II } & 87 & 51.8 & 71 & 81.6 & 16 & 18.4 & 0.1742 \\ \text { III-IV } & 81 & 48.2 & 59 & 72.8 & 22 & 27.2 & \end{array}$

$\begin{array}{llllllll}\text { Extranodal (IPI) } & & & & & & & \\ 1 \text { Site } & 92 & 54.8 & 72 & 78.3 & 20 & 21.7 & 0.7644 \\ >1 \text { Site } & 76 & 45.2 & 58 & 76.3 & 18 & 23.7 & \end{array}$

$L D H^{\mathrm{a}}(I P I)$

$\begin{array}{llllllll}\text { Normal }(<480) & 78 & 46.4 & 62 & 79.5 & 16 & 20.5 & 0.5427 \\ \text { High }(>480) & 90 & 53.6 & 68 & 75.6 & 22 & 24.4 & \end{array}$

$|P|^{b}$ group

$\begin{array}{lrllllll}\text { Low-low inter } & 110 & 65.5 & 87 & 79.1 & 23 & 20.9 & 0.4688 \\ \text { High inter-high } & 58 & 34.5 & 43 & 74.1 & 15 & 25.9 & \end{array}$

$\begin{array}{lrrrrrrr}G C \text { vs } A B C^{c} & & & & & & & \\ A B C & 199 & 79.3 & 138 & 69.4 & 61 & 30.6 & 0.0002 \\ G C & 52 & 20.7 & 48 & 92.3 & 4 & 7.7 & \end{array}$

$\begin{array}{llllllll}p-A K T^{\$} & & & & & & & \\ \text { High (2-3) } & 109 & 43.2 & 88 & 80.7 & 21 & 19.3 & 0.0274 \\ \text { Low (0-1) } & 143 & 56.8 & 98 & 68.5 & 45 & 31.5 & \end{array}$

$\begin{array}{lrrrrrrr}\text { PTEN }^{\text {\$ }} & & & & & & & \\ \text { Low (0-1) } & 88 & 34.9 & 57 & 64.8 & 31 & 35.2 & 0.0182 \\ \text { High (2-3) } & 164 & 65.1 & 129 & 78.7 & 35 & 21.3 & \end{array}$

Table 1 Continued

\begin{tabular}{|c|c|c|c|c|c|c|c|}
\hline \multirow{2}{*}{$\begin{array}{l}\text { DLBCL-301 } \\
\text { August } 2008\end{array}$} & \multicolumn{2}{|c|}{ Total } & \multicolumn{2}{|c|}{ Any-one-high } & \multicolumn{2}{|c|}{ Both low } & \multirow[t]{2}{*}{$P$-value } \\
\hline & No. & $\%$ & No. & $\%$ & No. & $\%$ & \\
\hline \multicolumn{8}{|l|}{ Ki-67 } \\
\hline$>50$ & 161 & 63.4 & 129 & 80.1 & 32 & 19.9 & 0.0012 \\
\hline$\leqslant 50$ & 93 & 36.6 & 57 & 61.3 & 36 & 38.7 & \\
\hline \multicolumn{8}{|l|}{ PhosphoGSK3 } \\
\hline$>0$ & 204 & 83.6 & 158 & 77.4 & 46 & 22.6 & 0.0047 \\
\hline$\leqslant 0$ & 40 & 16.4 & 22 & 55.0 & 18 & 45.0 & \\
\hline 5-year overall survival & & & & 76.2 & & 57.5 & 0.0028 \\
\hline
\end{tabular}

${ }^{\mathrm{a}} \mathrm{LDH}$ categorization is based on the range of normal values in the clinical laboratory at our institution.

$\mathrm{b}_{\mathrm{IPI}}$ information was available in only 156 patients.

${ }^{\mathrm{C}} \mathrm{GC}$ vs $A B C$, germinal center versus activated $B$ cell phenotype.

${ }^{\$}$ The analysis failure of some markers for these IHC markers was attributed to missing or nonrepresentative spots.

pathways that participate in growth, proliferation and survival of malignant cells. ${ }^{4,5,10}$ We sought to determine whether PHA665752 inhibits constitutive phosphorylation of c-Met in DLBCL cell lines. DLBCL cell lines were treated with 1 and $5 \mu \mathrm{M}$ PHA665752, cells were lysed and proteins were analyzed by western blot. As shown in Figure 3a, c-Met was found to be constitutively phosphorylated (p-Met) in SUDHL4, SUDHL5 and SUDH10 cell lines and treatment of these cell lines with PHA665752 dephosphorylated c-Met in all cell lines in a dose-dependent manner. In addition, AKT was also found to be constitutively activated in all three cell lines and PHA665752 treatment dephosphorylated AKT and its downstream targets, FOXO-1 and GSK-3 in DLBCL cell lines. These data suggest that PHA665752 treatment causes inactivation of c-Met, leading to inactivation of AKT and downstream signaling molecules FOXO1 and GSK3 in DLBCL cell lines.

To better understand the association between c-Met and AKT, we performed transfection studies with siRNA against c-Met and AKT. Expression of c-Met-specific siRNA in DLBCL cells downregulated c-Met expression and dephosphorylated AKT. However, AKT-specific siRNA transfection in SUDHL4 cell line did not alter c-Met expression, but dephosphorylated AKT (Figure 3b). In addition, PI3-kinase and AKT inhibitors decreased AKT phosphorylation without affecting c-Met phosphorylation level in SUDHL4 cells (data not shown). These data support our hypothesis that $\mathrm{c}-$ Met mediates AKT activation in DLBCL cell lines.

We further investigated whether HGF is responsible for activation of c-Met and AKT in DLBCL cell lines. Serumstarved DLBCL cells were stimulated with $100 \mathrm{ng} / \mathrm{ml}$ HGF for various time periods, as indicated. Cells were lysed and 
a

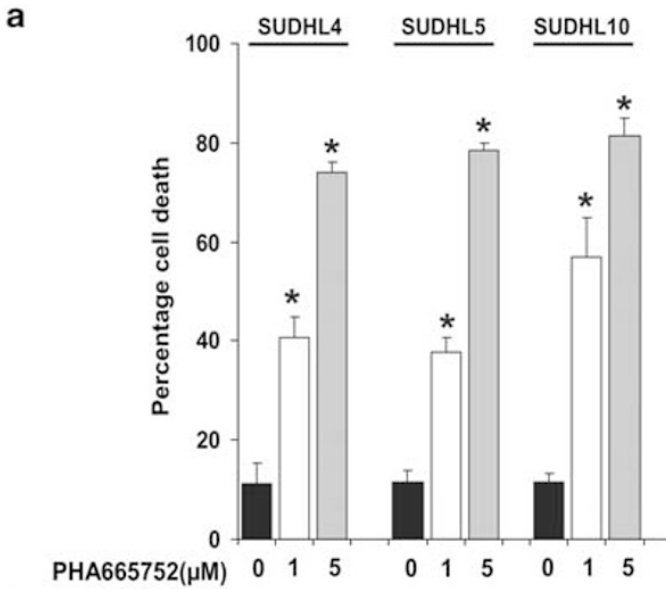

$c$

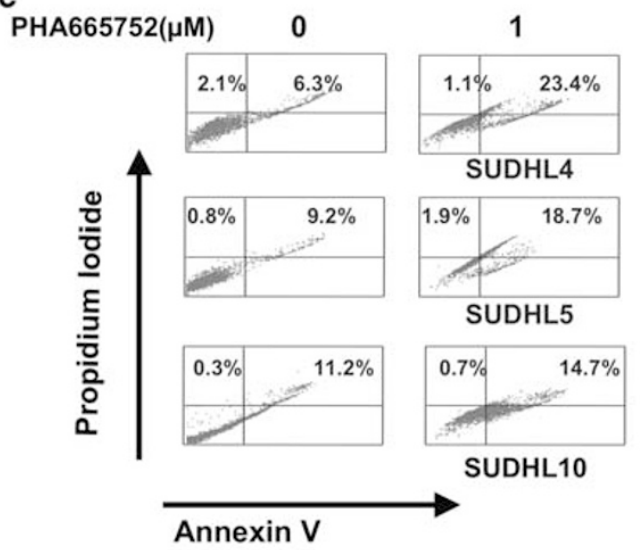

b PHA665752( $\mu \mathrm{M}) \quad 0$

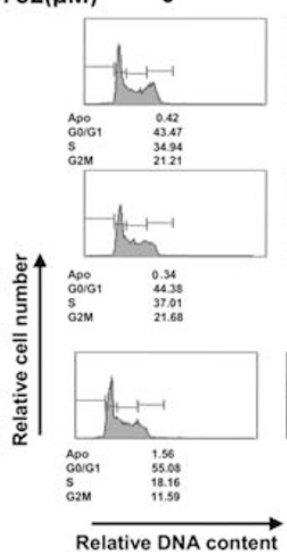

5
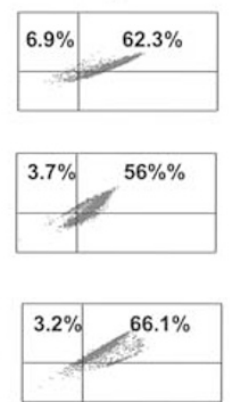

d
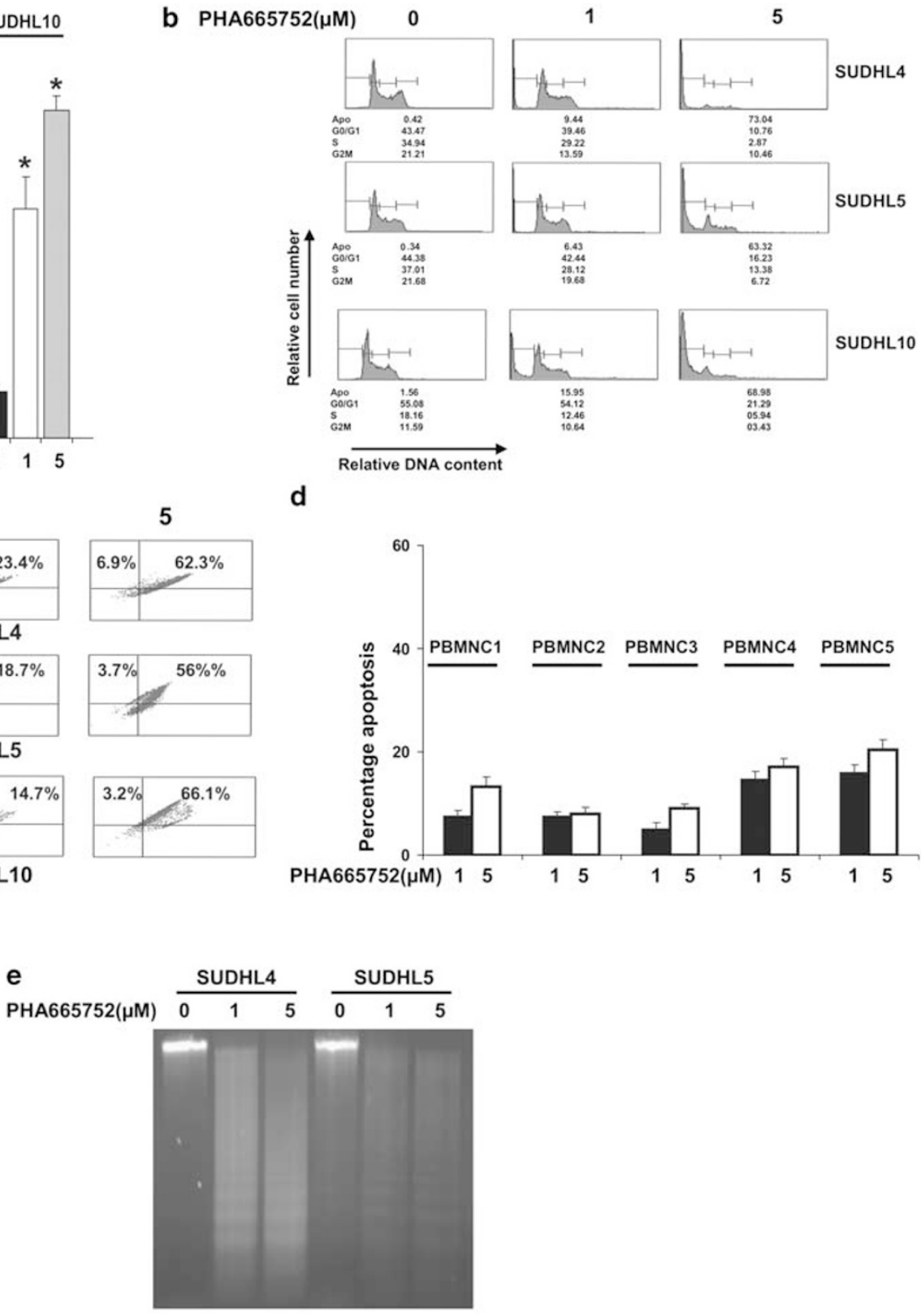

Figure 2 PHA665752 causes cell death of DLCBL cells in a dose-dependent manner. (a) SUDHL4, SUDHL5 and SUDHL10 cells were incubated with indicated doses of PHA665752 for $24 \mathrm{~h}$. Percentage cell death was scored using trypan blue exclusion dye. The graph displays the mean \pm s.d. (standard deviation) of three independent experiments. Asterisk denotes $P<0.001$, which is statistically significant (Student's $t$-test). (b) PHA665752 treatment increases sub-G1 (Apo) population DLBCL cell lines. SUDHL4, SUDHL 5 and SUDHL10 cells were treated with 1 and $5 \mu \mathrm{M}$ PHA665752 for $24 \mathrm{~h}$. Thereafter, the cells were washed and stained with propidium iodide, and analyzed for DNA content by flow cytometry, as described in Materials And Methods. A representative of four independent experiments is shown. (c) PHA665752 induced apoptosis in DLBCL cell lines. SUDHL4, SUDHL5 and SUDHL10 cells lines were treated with 1 and $5 \mu \mathrm{M}$ PHA665752 for $24 \mathrm{~h}$ and cells were subsequently stained with flourescein-conjugated annexin-V antibody and propidium iodide (PI) and ratio of apoptotic cells was analyzed by flow cytometry. A representative of three independent experiments is depicted in the figure. (d) PBMNCs from five healthy donors were treated with 1 and $5 \mu \mathrm{M}$ PHA665752 for $24 \mathrm{~h}$ and cells were subsequently stained with flourescein-conjugated annexin-V antibody and propidium iodide (PI) and ratio of apoptotic cells was analyzed by flow cytometry. The graph displays the mean \pm s.d. (standard deviation) of three independent experiments. (e) SUDHL4 and SUDHL5 cells were treated with 1 and $5 \mu \mathrm{M}$ PHA665752 for $24 \mathrm{~h}$, and DNA was extracted and separated by electrophoresis on $1.5 \%$ agarose gel.

proteins were analyzed by western blot. As shown in Figure 3c, HGF treatment induced c-Met phosphorylation as early as $5 \mathrm{~min}$ and remained phosphorylated till $120 \mathrm{~min}$. Similarly, HGF treatment caused time-dependent phosphorylation of
AKT in DLBCL cell lines (Figure 3c). Furthermore, pretreatment of DLBCL cell line with PHA665752 prevented HGF-mediated phosphorylation of c-Met and AKT (Figure 3d). However, pretreatment of SUDHL4 cell line with 

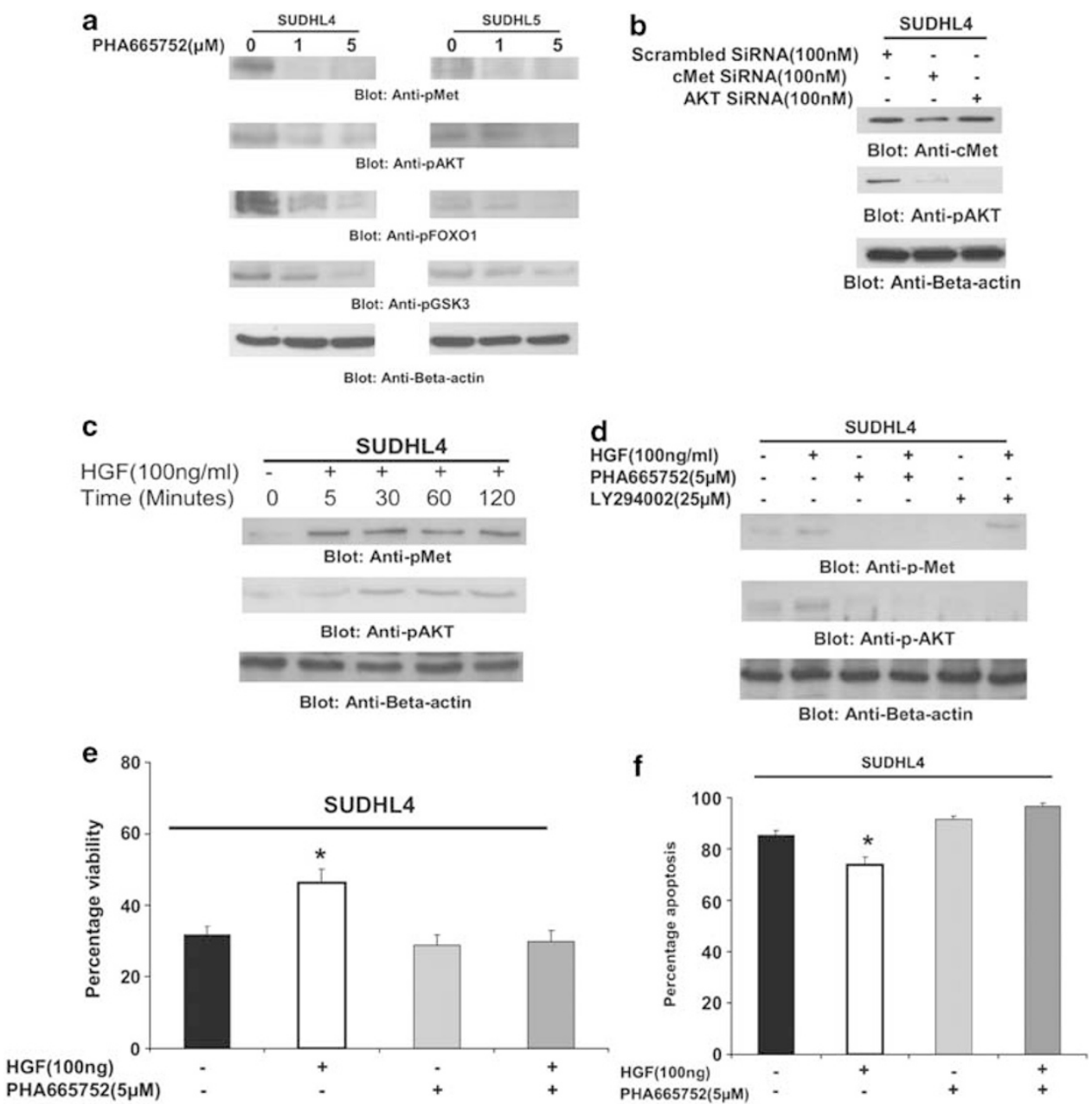

Figure 3 PHA665752 treatment causes dephosphorylation of constitutive p-Met and AKT and its substrates in DLBCL cell lines. (a) SUDHL4 and SUDHL5 cells were treated with 1 and $5 \mu \mathrm{M}$ PHA665752 for $24 \mathrm{~h}$. After cell lysis, equal amounts of proteins were separated by SDS-PAGE, transferred to immobilon membrane and immunoblotted with antibodies against p-Met, p-AKT, p-FOXO1, p-GSK3 and $\beta$-actin, as indicated. (b) c-Met siRNA dephosphorylates AKT in DLBCL cell lines. SUDHL4 cells were transfected with $100 \mathrm{nM}$ scrambled siRNA, $100 \mathrm{nM}$ c-Met and $100 \mathrm{nM}$ AKT siRNA with Lipofectamine, as described in Materials and Methods. After $48 \mathrm{~h}$ of transfection, cells were lysed and equal amounts of proteins were separated by SDS-PAGE, transferred to immobilon membrane and immunoblotted with antibodies against, c-Met, p-AKT and $\beta$-actin, as indicated. (c) HGF-mediated phosphorylation of c-Met and AKT in DLBCL cells. SUDHL4 cells were serum starved for $24 \mathrm{~h}$ and then treated with $100 \mathrm{nM}$ HGF for various time periods as indicated. After cell lysis, equal amounts of proteins were separated by SDS-PAGE, transferred to immobilon membrane and immunoblotted with antibodies against p-Met, p-AKT and $\beta$-actin as indicated. (d) PHA665752 inhibition inactivates HGF-induced c-Met and AKT in DLBCL cells. SUDHL4 cell line was serum starved for $24 \mathrm{~h}$ and treated with and without $5 \mu \mathrm{M}$ PHA665752 and $25 \mu \mathrm{M} \mathrm{LY} 294002$ for $12 \mathrm{~h}$ in the presence and absence of $100 \mathrm{nM}$ HGF. After treatment, cells were lysed and equal amounts of proteins were separated by SDS-PAGE, transferred to immobilon membrane and immunoblotted with antibodies against, c-Met, $\mathrm{p}$-AKT and $\beta$-actin as indicated. (e) HGF protects DLBCL cells from serum starvation-induced inhibition of cell viability. SUDHL4 cells were serum starved for $24 \mathrm{~h}$ and treated with $100 \mathrm{ng} / \mathrm{ml} \mathrm{HGF}, 5 \mu \mathrm{M}$ PHA665752 or a combination of both for $24 \mathrm{~h}$. Cell proliferation assays were performed using MTT and (f) apoptosis was measured using annexin-V/PI dual staining as described in Materials and Methods. The graph displays the mean \pm s.d. (standard deviation) of three independent experiments. ${ }^{\star} P<0.05$, statistically significant (Students $t$-test).

LY294002 failed to in-activate HGF-mediated phosphorylation of c-Met, even though AKT was inactivated (Figure 3d). These results clearly showed that c-Met mediated activation of AKT in DLBCL cell lines.

To further assess the role of HGF on DLBCL cells, we serum starved SUDHL4 cells for $24 \mathrm{~h}$ and then incubated with $100 \mathrm{ng}$ of HGF or $5 \mu \mathrm{M}$ of PHA665752, either alone or in combination for $24 \mathrm{~h}$, and cells were assessed for cell viability by MTT assay and apoptosis by annexin-V/PI dual staining. As shown in Figure 3e, serum starvation caused inhibition of cell viability in SUDHL4 cells, on the other hand, addition of $100 \mathrm{ng}$ HGF significantly protected these cells from serum starved inhibition of viability $(P=0.048)$. HGF stimulation however failed to protect serum-starved SUDHL4 cells from PHA665752-induced cell inhibition. Furthermore, HGF also significantly protected SUDHL4 cells from serum starvation-induced apoptosis $(P=0.012)$ (Figure 3f). Interestingly, HGF treatment of serum-starved SUDHL4 cells failed to protect from apoptosis after PHA665752 treatment (Figure 3f). These data indicated that 
HGF-mediated activation of c-Met had an important role in DLBCL cell proliferation and inhibition of apoptosis.

\section{PHA665752-Induces Apoptosis by Activation of Bax Through Activation of Mitochondrial Pathway in DLBCL Cells}

To detect whether inactivation of AKT led to apoptosis of DLBCL cells through activation of intrinsic pathway, we examined the activation of Bax in response to PHA665752 treatment. SUDHL5 cells were treated with $5.0 \mu \mathrm{M}$ PHA665752 for various time periods. Cells were lysed with $1.0 \%$ Chaps lysis buffer and lysates were immunoprecipitated with Bax 6A7 antibody that recognizes only the conformationally changed Bax protein. The detergent Chaps has been shown to retain the Bax protein in its native conformation. As shown in Figure 4a, conformationally changed Bax was detected within $2 \mathrm{~h}$, peaked at $8 \mathrm{~h}$ and started decreasing after $8 \mathrm{~h}$ of treatment with $5 \mu \mathrm{M}$ PHA665752 in SUDHL5 cell line. The conformational changes in Bax have previously been shown to occur only in apoptotic cells, possibly having a role in mediating cytochrome $c$ release. ${ }^{25,27}$ Similar data were obtained for other DLBCL cell lines (data not shown).

\section{PHA665752 Treatment of DLBCL Cells Induces Loss of Mitochondrial Membrane Potential and Subsequently Release of Cytochrome $\boldsymbol{c}$ to Cytosol}

We further tested the effect of PHA665752 on mitochondrial membrane potential using JC1 dye. SUDHL4, SUDHL5 and SUDHL10 cells were treated with 1 and $5.0 \mu \mathrm{M}$ PHA665752 for $24 \mathrm{~h}$. As shown in Figure 4b, PHA665752 treatment of these cells resulted in loss of mitochondrial membrane potential, as measured by JC1-stained green florescencedepicting apoptotic cells. Cytochrome $c$ release from mitochondria in SUDHL5 cells treated with PHA665752 was examined by western blot analysis. Cells were treated with 1 and $5.0 \mu \mathrm{M}$ concentration of PHA665752 for $24 \mathrm{~h}$. Cytosolspecific and mitochondria-free, as well as mitochondrial lysates was prepared as described in Materials and Methods. Cell fractionation results showed that cytochrome $c$ translocated from mitochondria to cytosol after PHA665752 treatment. As shown in Figure 4c, the protein levels of cytochrome $c$ increased in the cytosolic fractions and, concomitantly, decreased in mitochondria-enriched heavy membrane fractions of SUDHL5 cells after PHA665752 treatment. These results suggest that inhibition of c-Met activity in DLBCL cells causes apoptosis through the release of cytochrome $c$ from the mitochondria. These results further support our notion that PHA665752-induced apoptosis in DLBCL cells involve signaling at the mitochondrial level.

\section{PHA665752-Induced Apoptosis Occurs Through Activation of Caspases in DLBCL Cells}

As caspases are important mediators of apoptosis in response to various apoptotic stimuli, ${ }^{24-26}$ we investigated whether a
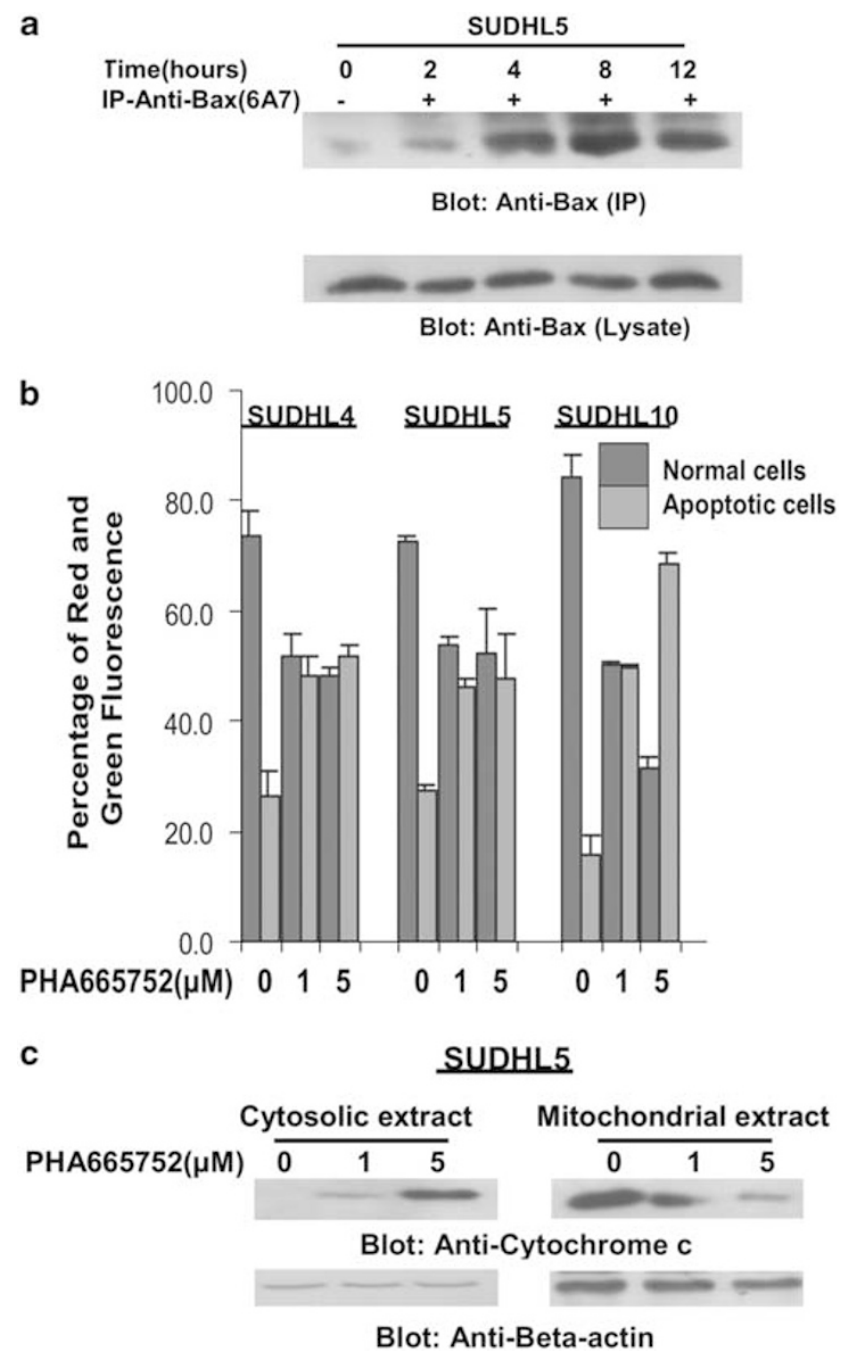

Figure 4 PHA665752-induced mitochondrial apoptotic pathway in DLBCL cells. (a) c-Met-induced Bax activation in DLBCL cells. SUDHL5 cells were treated with $5 \mu \mathrm{M}$ PHA665752 for 2, 4, 8 and $12 \mathrm{~h}$, as indicated, lysed in $1 \%$ Chaps lysis buffer and subjected to immunoprecipitation (IP) with anti-Bax 6 A7 antibody. Immunoprecipitated Bax was detected by immunoblotting with Bax antibody. In addition, the total cell lysates were applied directly to SDS-PAGE, transferred to immobilon membrane and immunoblotted with specific anti-Bax polyclonal antibody. (b) Loss of mitochondrial potential by PHA665752 treatment of DLBCL cells. SUDHL4, SUDHL 5 and SUDHL10 cells were treated with and without 1 and $5 \mu \mathrm{M}$ PHA665752 for $24 \mathrm{~h}$. Live cells with intact mitochondrial membrane potential (red bars) and dead cells with lost mitochondrial membrane potential (green bars) were measured by JC1 staining and analyzed by flow cytometry as described in Materials and Methods. An average of three independent experiments is depicted in the figure. (c) PHA665752-induced release of cytochrome c. SUDHL5 cells were treated with and without 1 and $5 \mu \mathrm{M}$ PHA665752 for $24 \mathrm{~h}$. Mitochondrial-free cytosolic fractions, as well as mitochondrial extracts, were isolated as described in Materials and Methods. Cell extracts were separated on SDS-PAGE, transferred to PVDF membrane and immunoblotted with an antibody against cytochrome $c$. $\beta$-Actin was used for equal loading.

PHA665752 treatment also caused their activation. SUDHL4 and SUDHL5 cells were treated with 1.0 and $5.0 \mu \mathrm{M}$ PHA665752 for $24 \mathrm{~h}$ and immunoblotted with caspase-9, 


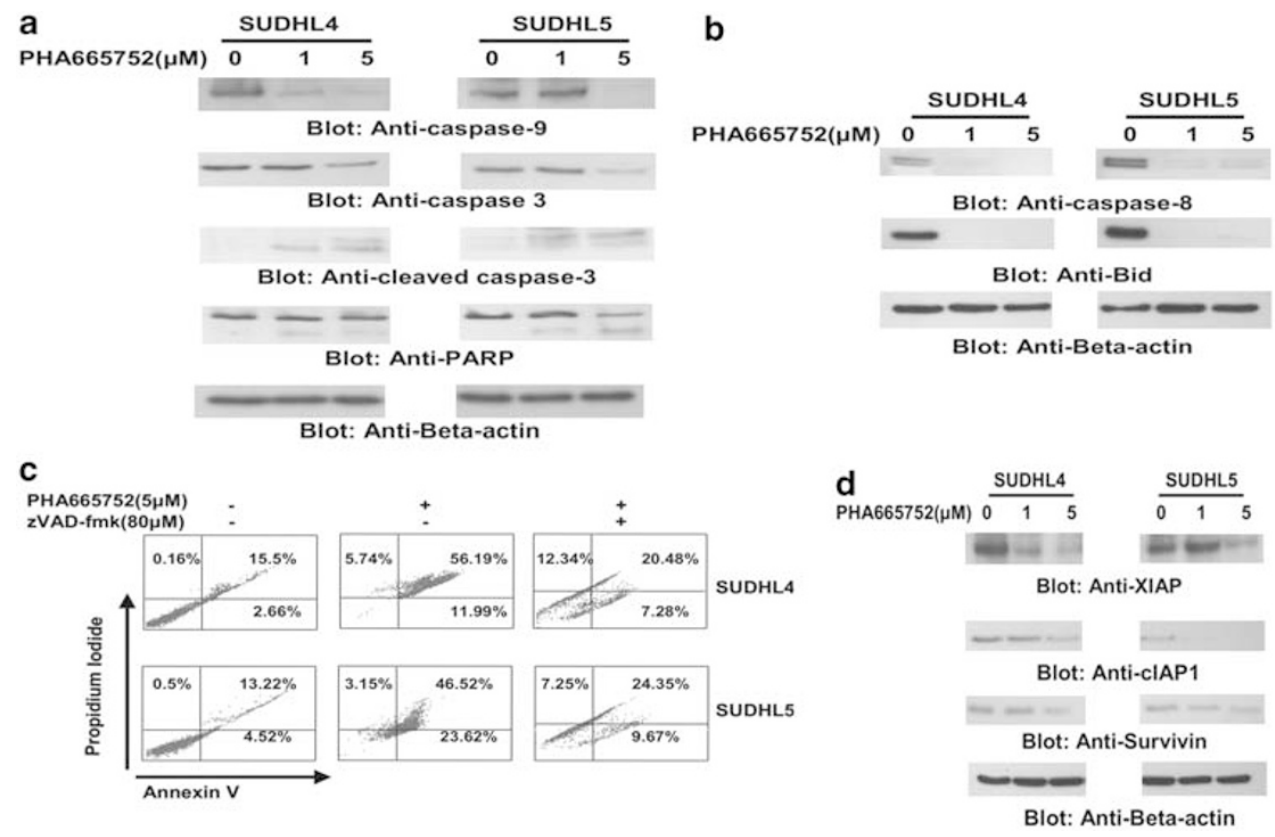

Figure 5 (a) Activation of caspases induced by treatment of PHA665752 in DLBCL cells. SUDHL4 and SUDHL5 cells were treated with and without 1 and $5 \mu \mathrm{M}$ PHA665752 for $24 \mathrm{~h}$. Cells were lysed and proteins were immunoblotted with antibodies against caspase-9, pro-caspase-3, cleaved caspase-3, PARP, and beta-actin. Each experiment was repeated three times to confirm reproducibility. (b) SUDHL4 and SUDHL5 cells were treated with and without 1 and $5 \mu \mathrm{M}$ PHA665752 for $24 \mathrm{~h}$. Cells were lysed and proteins were immunoblotted with antibodies against caspase-8, BID and beta-actin. (c) SUDHL4 and SUDHL5 cell lines were pre-treated with either $80 \mu \mathrm{M} z-V A D / f m k$ for 2 hrs and treated with $50 \mu \mathrm{M}$ of C-75 for $24 \mathrm{~h}$ and apoptosis was measured by annexinV/PI staining. (d) PHA665752-induced downregulation of CIAP1, XIAP and Survivin expression. SUDHL4 and SUDHL5 cells were treated with and without 25 and $50 \mu \mathrm{M} \mathrm{C-75}$ for $24 \mathrm{~h}$. Cells were lysed and equal amount of proteins were separated on SDS-PAGE, transferred to PVDF membrane, and immunoblotted with antibodies against XIAP, cIAP1, Survivin, and beta-actin as indicated.

caspase-3, cleaved caspase-3 and PARP antibodies. As shown in Figure 5a, PHA665752 treatment of DLBCL cells induced activation of caspase- 9 and caspase- 3 cleavage in both cell lines. PARP, a downstream target of caspase-3, was also cleaved in both the cell lines, a hallmark of cells undergoing apoptosis. It has been shown by us and others that caspase-3 has the ability to activate caspase- 8 and cleave Bid, downstream of the mitochondrial pathway to potentate the apoptotic signal. ${ }^{31}$ Therefore, we sought to determine the expression of caspase-8 and Bid after treatment with PHA665752. SUDHL4 and SUDHL5 cells were treated with 1.0 and $5.0 \mu \mathrm{M}$ PHA665752 for $24 \mathrm{~h}$, cell lysates were separated on SDS-PAGE and immunoblotted with an antibody against caspase- 8 and Bid. Figure 5b shows that PHA665752 treatment resulted in reduction in the intensity of the fulllength band of pro-caspase- 8 indicating activation of caspase-8. Bid is a BH3-proapoptotic protein that can be cleaved directly by caspase 8 during apoptosis. The cleaved or truncated Bid also has a role in the induction of Bax conformational change and subsequent translocation to mitochondria. $^{32,33}$ Therefore, we examined the role of Bid cleavage after PHA665752 treatment of DLBCL cells. As shown in Figure 5b, treatment with PHA665752 resulted in decreased level of full-length of Bid. Furthermore, pretreatment of DLBCL cells with $80 \mu \mathrm{M} \mathrm{z}$-VAD-fmk, a universal inhibitor of caspases, abrogated caspase- 3 activation (data not shown) and prevented apoptosis (Figure 5c) induced by PHA665752, clearly indicating that caspases have a critical role in PHA665752-induced apoptosis in DLBCL cells.

\section{Modulation of IAP Protein Family During PHA665752-Induced Apoptosis in DLBCL Cells}

PI3-kinase/AKT pathway has been shown to regulate the stabilization of XIAP. ${ }^{17,27}$ As our data showed that c-Met signal occurs through AKT, we therefore examined whether PHA665752 treatment induced apoptosis by modulating the expression of XIAP and other members of IAP family. DLBCL cells were treated with 1 and $5 \mu \mathrm{M}$ PHA665752 for $24 \mathrm{~h}$, and expression of XIAP, cIAP1 and Survivin were determined using western blotting. As shown in Figure 5d, PHA665752 treatment caused dose-dependent downregulation of XIAP, cIAP1 and Survivin in SUDHL4 and SUDHL 5 cells. These results indicate that IAP proteins may also be involved in PHA665752-induced apoptosis.

\section{DISCUSSION}

The HGF receptor, c-Met, is a receptor tyrosine kinase receptor that has been shown to be oncogenic in variety of tumors. ${ }^{34,35} \mathrm{HGF} / \mathrm{c}-\mathrm{Met}$ signaling pathway is known to activate AKT for its survival and antiapoptotic functions. ${ }^{10,36}$ In light of these findings, we investigated c-Met overexpression in a large cohort of Middle Eastern DLBCL 
patient samples, and its association with AKT and its signaling partners. Our data showed that c-Met was overexpressed in $73.2 \%$ DLBCL samples and significantly associated with p-AKT and p-GSK3. c-Met overexpression was significantly associated with $\mathrm{Ki}-67(P=0.0012)$ and was significantly more common in the GC. Intriguingly, overexpression of c-Met in DLBCL was associated with better survival $(P=0.0028)$ and remained significant in multivariate analysis with IPI, thereby confirming $\mathrm{c}$-Met as independent prognostic marker for better outcome in DLBCL. A similar finding that c-Met expression is associated with a favorable prognostic factor in invasive breast carcinoma has been reported. ${ }^{37}$ These results contradict the finding of an earlier study in which c-MET overexpression has been found to be associated with a worse outcome in DLBCL. ${ }^{14}$ There are several possible reasons for better outcome due to overexpression of c-Met in Saudi DLBCL patients. One speculation could be that lymphoma cells with c-Met have retained the physiological growth control by c-Met, which is supported by the strong link between c-Met and GC subtype DLBCL. Another factor that can contribute to the favorable outcome in DLBCL-expressing c-Met is the significant association with high proliferative index, Ki67 $(P=0.0012)$ that might result in cell cycle progression and possibly chemotherapy sensitivity. Interestingly, previous studies in other NHLs such as follicular lymphoma have shown that tumors with more proliferating cells respond better to chemotherapy and have better survival. ${ }^{38,39}$ Furthermore, multifunctional effects of c-Met signaling has been shown to depend on the in vivo circumstances, HGF concentrations, tumor cell differentiation, including number, type and quality of intercellular junctions and finally on a delicate balance between epithelial/ lymphoid and mesenchymal compartments. ${ }^{40,41}$ Thus, difference in the sample size, ethnic origin, using different methodology of immunohistochemical analysis (TMA vs large sections) and different scoring system might also be contributing factors.

Our in vitro experiments with a panel of DLBCL cell lines that express p-Met showed that inhibition of c-MET tyrosine kinase activity by PHA665752, dephosphorylated AKT and its downstream substrates FOXO1 and GSK3 and induced apoptosis, which further indicated that overexpression of c-MET can modulate AKT activity. Furthermore, gene silencing studies using siRNA specific for c-Met depleted expression of c-Met as well as abrogated c-Met-mediated AKT signaling. On the other hand, AKT-specific siRNA did not affect c-Met expression, indicating that c-Met signal through AKT in DLBCL cell lines. These data strongly suggest that the activity of PI3K/AKT pathway is partly driven by the basal c-Met activity. In addition, expression of c-Met was not regulated by PI3K/AKT pathway, as inhibition of AKT did not induce any c-Met loss. This hypothesis is supported by our data that show that c-Met inhibition prevents cell proliferation and induces mitochondrial and caspasedependent apoptosis in DLBCL cell lines. Furthermore, our data also show that inhibition of c-Met cause inactivation of AKT, resulting in downregulation of IAPs that cause activation of caspase-dependent apoptosis in DLBCL cells. Interestingly, PHA665752 failed to induce apoptosis in normal PBMNCs owing to lack of c-Met expression. This data also suggest highly selective targeting of tumor cells with high c-Met expression and sparing of normal cells, thereby avoiding potential systemic side effects.

In summary, high prevalence of c-Met deregulation was seen in large Saudi cohort of DLBCL patients, and c-Met overexpression was a prognostic marker for better survival for Middle Eastern DLBCL patients. c-Met overexpression was significantly associated with activated AKT, Ki-67 and was more common in the GC. Further, our in vitro data suggest that inhibition of c-Met activity by PHA665752 induces apoptosis in DLBCL cells through inhibition of PI3K/ AKT pathway and activation of the caspases.

Supplementary Information accompanies the paper on the Laboratory Investigation website (http://www.laboratoryinvestigation.org)

\section{DISCLOSURE/CONFLICT OF INTEREST}

The authors declare no conflict of interest.

1. The Non-Hodgkin's Lymphoma Classification Project. A clinical evaluation of the International Lymphoma Study Group classification of non-Hodgkin's lymphoma. Blood 1997;89:3909-3916.

2. Muris JJ, Cillessen SA, Vos W, et al. Immunohistochemical profiling of caspase signaling pathways predicts clinical response to chemotherapy in primary nodal diffuse large B-cell lymphomas. Blood 2005;105:2916-2923.

3. Hartge $P$, Wang SS. Overview of the etiology and epidemiology of lymphoma. In: Mauch PM, Armitage JO, Coiffier B, Dalla-Favera R, Harris NL (eds). Non-Hodgkin's Lymphomas. Lippincott, Williams and Wilkins: New York, NY, 2004, pp 711-727.

4. van der Voort R, Taher TE, Derksen PW, et al. The hepatocyte growth factor/Met pathway in development, tumorigenesis, and B-cell differentiation. Adv Cancer Res 2000;79:39-90.

5. van der Voort R, Taher TE, Keehnen RM, et al. Paracrine regulation of germinal center $B$ cell adhesion through the c-met-hepatocyte growth factor/scatter factor pathway. J Exp Med 1997;185:2121-2131.

6. Christensen JG, Burrows J, Salgia R. c-Met as a target for human cancer and characterization of inhibitors for therapeutic intervention. Cancer Lett 2005;225:1-26.

7. Tarte K, Zhan F, De Vos J, et al. Gene expression profiling of plasma cells and plasmablasts: toward a better understanding of the late stages of B-cell differentiation. Blood 2003;02:592-600.

8. Capello D, Gaidano G, Gallicchio M, et al. The tyrosine kinase receptor met and its ligand HGF are co-expressed and functionally active in HHV-8 positive primary effusion lymphoma. Leukemia 2000;14: 285-291.

9. Borset M, Hjorth-Hansen $\mathrm{H}$, Seidel $\mathrm{C}$, et al. Hepatocyte growth factor and its receptor c-met in multiple myeloma. Blood 1996;88:3998-4004.

10. Tjin EP, Groen RW, Vogelzang I, et al. Functional analysis of HGF/MET signaling and aberrant HGF-activator expression in diffuse large B-cell lymphoma. Blood 2006;107:760-768.

11. Hsiao LT, Lin JT, Yu IT, et al. High serum hepatocyte growth factor level in patients with non-Hodgkin's lymphoma. Eur J Haematol 2003;70: 282-289.

12. Giles FJ, Vose JM, Do KA, et al. Clinical relevance of circulating angiogenic factors in patients with non-Hodgkin's lymphoma or Hodgkin's lymphoma. Leuk Res 2004;28:595-604.

13. Kawano $R$, Ohshima $K$, Karube $K$, et al. Prognostic significance of hepatocyte growth factor and c-MET expression in patients with diffuse large B-cell lymphoma. Br J Haematol 2004;127:305-307. 
14. Abubaker J, Jehan Z, Bavi P, et al. Clinicopathological analysis of papillary thyroid cancer with PIK3CA alterations in a Middle Eastern population. J Clin Endocrinol Metab 2008;93:611-618.

15. Jaffe ES, Harris NL, Stein H, et al. Pathology and Genetics of Tumors of Hematopoetic and Lymphoid Tissues: World Health Organization Classification of Tumors. IARC Press: Lyon, France, 2001.

16. Bavi $P$, Jehan $Z$, Atizado V, et al. Prevalence of fragile histidine triad expression in tumors from Saudi Arabia: a tissue microarray analysis. Cancer Epidemiol Biomarkers Prev 2006;15:1708-1718.

17. Uddin S, Hussain A, Al-Hussein K, et al. Inhibition of phosphatidylinositol $3^{\prime}$-kinase induces preferentially killing of PTEN-null T leukemias through AKT pathway. Biochem Biophys Res Commun 2004;320:932-938.

18. McCarty Jr KS, Miller LS, Cox EB, et al. Estrogen receptor analyses. Correlation of biochemical and immunohistochemical methods using monoclonal antireceptor antibodies. Arch Pathol Lab Med 1985;109: 716-721.

19. Uddin $S$, Hussain AR, Siraj $A K$, et al. Role of phosphatidylinosito $3^{\prime}$-kinase/AKT pathway in diffuse large B-cell lymphoma survival. Blood 2006;108:4178-4186.

20. Abubaker J, Bavi P, Al-Harbi S, et al. Clinicopathological analysis of colorectal cancers with PIK3CA mutations in Middle Eastern population. Oncogene 2008;27:3539-3545.

21. Hans $C P$, Weisenburger DD, Greiner TC, et al. Confirmation of the molecular classification of diffuse large B-cell lymphoma by immunohistochemistry using a tissue microarray. Blood 2004;103:275-282.

22. Bavi P, Abubaker J, Hussain A, et al. Reduced or absent cyclin $H$ expression is an independent prognostic marker for poor outcome in diffuse large B-cell lymphoma. Hum Pathol 2008;39:885-894.

23. Camp RL, Dolled-Filhart M, Rimm DL. X-tile: a new bio-informatics too for biomarker assessment and outcome-based cut-point optimization. Clin Cancer Res 2004;10:7252-7259.

24. Hussain AR, Al-Rasheed M, Manogaran PS, et al. Curcumin induces apoptosis via inhibition of $\mathrm{PI} 3^{\prime}$-kinase/AKT pathway in acute $\mathrm{T}$ cell leukemias. Apoptosis 2006;11:245-254.

25. Hussain AR, Al-Jomah NA, Siraj AK, et al. Sanguinarine-dependent induction of apoptosis in primary effusion lymphoma cells. Cancer Res 2007:67:3888-3897.

26. Uddin S, Hussain AR, Manogaran PS, et al. Curcumin suppresses growth and induces apoptosis in primary effusion lymphoma. Oncogene 2005;24:7022-7030.

27. Hussain AR, Ahmed $\mathrm{M}$, Al-Jomah NA, et al. Curcumin suppresses constitutive activation of nuclear factor-kappa $B$ and requires functional Bax to induce apoptosis in Burkitt's lymphoma cell lines. Mol Cancer Ther 2008;7:3318-3329.
28. Uddin S, Ah-Kang J, Ulaszek J, et al. Differentiation stage-specific activation of p38 mitogen-activated protein kinase isoforms in primary human erythroid cells. Proc Natl Acad Sci USA 2004;101:147-152.

29. Uddin S, Hussain AR, Al-Hussein KA, et al. Inhibition of phosphatidylinositol 3'-kinase/AKT signaling promotes apoptosis of primary effusion lymphoma cells. Clin Cancer Res 2005;11:3102-3108.

30. Uddin S, Bu R, Ahmed $\mathrm{M}$, et al. Overexpression of leptin receptor predicts an unfavorable outcome in Middle Eastern ovarian cancer. Mol Cancer 2009;8:74

31. Uddin S, Siraj AK, Al-Rasheed M, et al. Fatty acid synthase and AKT pathway signaling in a subset of papillary thyroid cancers. J Clin Endocrinol Metab 2008;93:4088-4097.

32. Uddin S, Ahmed M, Bavi P, et al. Bortezomib (Velcade) induces p27Kip1 expression through S-phase kinase protein 2 degradation in colorectal cancer. Cancer Res 2008;68:3379-3388.

33. Hussain AR, Ahmed M, Ahmed SO, et al. Proteasome inhibitor MG-132 mediated expression of p27Kip1 via S-phase kinase protein 2 degradation induces cell cycle coupled apoptosis in primary effusion lymphoma cells. Leuk Lymphoma 2009;50:1204-1213.

34. Börset $M$, Hjorth-Hansen $H$, Seidel $C$, et al. Hepatocyte growth factor and its receptor c-met in multiple myeloma. Blood 1996;88:3998-4004.

35. Capello D, Gaidano G, Gallicchio M, et al. The tyrosine kinase recepto met and its ligand HGF are co-expressed and functionally active in HHV-8 positive primary effusion lymphoma. Leukemia 2000;14: 285-291.

36. Watson GA, Zhang X, Stang MT, et al. Inhibition of c-Met as a therapeutic strategy for esophageal adenocarcinoma. Neoplasia 2006;8:949-955.

37. Nakopoulou L, Gakiopoulou H, Keramopoulos A, et al. c-met tyrosine kinase receptor expression is associated with abnormal beta-catenin expression and favourable prognostic factors in invasive breast carcinoma. Histopathology 2000;36:313-325.

38. Björck E, Ek S, Landgren $\mathrm{O}$, et al. High expression of cyclin B1 predicts a favorable outcome in patients with follicular lymphoma. Blood 2005;105:2908-2915.

39. Wilson $\mathrm{WH}$, Teruya-Feldstein J, Fest $\mathrm{T}$, et al. Relationship of p53, bcl-2, and tumor proliferation to clinical drug resistance in non-Hodgkin's lymphomas. Blood 1997;89:601-609.

40. Seslar SP, Nakamura T, Byers SW. Regulation of fibroblast hepatocyte growth factor/scatter factor expression by human breast carcinoma cell lines and peptide growth factors. Cancer Res 1993;53: 1233-1238.

41. Rosen EM, Nigam SK, Goldberg ID. Scatter factor and the c-met receptor: a paradigm for mesenchymal/epithelial interaction. J Cell Biol $1994 ; 127: 1783-1787$ 